ÉGYPTE monde arabe

\section{Égypte/Monde arabe}

9 | 2012

Gouvernance locale dans le monde arabe et en

Méditerranée : Quel rôle pour les femmes?

\title{
Les femmes et les espaces publics à Téhéran
}

Women and public spaces in Teheran

\section{Mina Saidi Shahrouz}

\section{(2) OpenEdition}

Journals

Édition électronique

URL : https://journals.openedition.org/ema/2996

DOI : 10.4000/ema.2996

ISSN : 2090-7273

Éditeur

CEDEJ - Centre d'études et de documentation économiques juridiques et sociales

Édition imprimée

Date de publication : 1 janvier 2012

Pagination : 57-69

ISBN : 2 905838-48-5

ISSN : 1110-5097

Référence électronique

Mina Saidi Shahrouz, "Les femmes et les espaces publics à Téhéran », Égypte/Monde arabe [En ligne],

9 | 2012, mis en ligne le 31 décembre 2012, consulté le 07 juillet 2022. URL : http://

journals.openedition.org/ema/2996; DOI : https://doi.org/10.4000/ema.2996 


\section{Mina SaIdi Sharouz}

\section{RÉSUMÉ / ABSTRACT}

\section{LES FEMMES ET LES ESPACES PUBLICS A TÉHÉRAN}

Nous interrogeons, à travers cette recherche, le sens de la féminisation des espaces publics en Iran, un pays où les femmes sont supposé rester dans l'espace privé et sous le contrôle des hommes et des lois islamiques. Quels impacts peut avoir cette présence massive sur leur statut? Quelle place est réservée réellement aux femmes dans l'espace public ? Comment parviennent-elles à détourner les interdits et à utiliser les possibilités offertes par les politiques urbaines en leur faveur pour accéder à l'espace public ?

Nous examinerons les évolutions de cette appropriation féminine des espaces urbains à Téhéran à travers trois périodes : la période traditionnelle jusqu'au début de $20^{\text {e }}$ siècle, la modernisation "forcée " des rois Pahlavi et les bouleversements entraînés par la République Islamique. Cette étude historique nous facilite la lecture de la situation actuelle, marquée par l'irruption des femmes dans les espaces extérieurs. Cette nouvelle donne de la société urbaine prend racine dans les années 60 , où la visibilité dans l'espace urbain était réservée aux élites, et puis à partir de la révolution iranienne de 1979, qui a été décisive pour les femmes. En effet, en les contraignant au port du voile et en instituant une division sexuée de l'espace, la République Islamique semble avoir nourri un paradoxe en matière de mobilité des femmes. Nous démontrons, à travers des données sur les mobilités quotidiennes et des exemples d'usage de certains lieux publics, comment les femmes ont su transformer un devoir d'être dehors (sur l'appel des autorités pour manifester) en un certain droit à la ville.

\section{WOMEN AND PUBLIC SPACES IN TEHERAN}

We examine the evolutions of this feminine appropriation of urban spaces in Tehran through three periods. The traditional period, lasting until the beginning of the $20^{\text {th }}$ century, the "forced" modernization of the Pahlavi dynasty, and the upheavals of the Islamic republic. This historical survey facilitates our reading of the actual situation marked by the eruption of women in exterior spaces. This new use of the urban space took root in the 60s when visibility in the urban space 
was reserved for elites, and then in the Iranian revolution of 1979, which was decisive for women. In effect, by obliging them to wear the veil and by instituting a gender division of lived space, the Islamic republic seems to have nourished a paradox in terms of the mobility of women. We demonstrate through information on daily mobility and examples of their use of certain public spaces, how women knew how to transform an obligation to be in public (given by authorities in order to manifest) into a certain right to the city.

MINA SAIDI SHAROUZ est chercheur et responsable de l'Observatoire Urbain de Téhéran et des Villes d'Iran (O.U.T.I.) au sein de I'Institut Français de Recherche en Iran.

MINA SAIDI SHAROUZ is a researcher and the Director of the Urban Observatory of Teheran and Iranian cities (O.U.T.I) in the French Research Institute in Iran. 
Mina Saidi Sharouz

\section{LES FEMMES ET LES ESPACES PUBLICS A TÉHÉRAN}

$\mathrm{B}$ ien que le statut des femmes impose de lourdes contraintes, on constate que les femmes d'Iran n'ont jamais autant participé à la vie sociale que maintenant. Alors que les femmes étaient absentes des rues des villes islamiques traditionnelles, elles sont désormais présentes dans les rues de Téhéran. Qui sont-elles ? Où vontelles? Que font-elles? Ces questions ont rarement été posées, sinon de façon globale, à l'échelle de l'Iran tout entier, sans toujours prendre en compte la complexité de ce nouveau fait urbain en travaillant à l'échelle des catégories sociales ou culturelles, à l'échelle des quartiers.

Cet article a pour objectif de présenter les résultats d'une enquête de mobilités auprès des femmes de Téhéran en 2007/2008, permettant de mieux comprendre, à travers leurs pratiques et leur perception de l'espace, la place des femmes dans la société urbaine iranienne d'aujourd'hui. En examinant les caractéristiques et les modalités des mobilités quotidiennes à partir des données scientifiques, cette recherche contribue à dépasser les débats idéologiques autour des femmes en Iran et à établir une typologie de femmes par rapport à leurs mobilités et leur présence dans l'espace public.

\section{DE LA CITÉ «SANS FEMMES » AUX FEMMES DANS LA VILLE}

L'organisation des espaces en Iran repose sur la séparation des hommes et des femmes, et ceci à toutes les échelles spatiales. Si la cité traditionnelle s'est formée autour des lieux symboliques comme la mosquée, la citadelle (arg) et le bazar, ces tissus urbains, qui constituent sa chair, sont fortement sexués et ont pour fonction, d'éviter ou de permettre - sous conditions - la rencontre du masculin et du féminin. L'espace a toujours été inégalement réparti entre les femmes et les hommes. Les femmes utilisent une partie seulement de la maison et les hommes toute la ville. 
A l'origine de cette séparation le "nâmus" (honneur personnel...), cette notion, ancrée profondément dans la culture musulmane, définit des comportements et des modes de vie spécifiques et sert à assurer la pureté sexuelle. La femme fait partie du patrimoine de l'homme au même titre que sa maison, sa ville et même son pays (on défend son pays comme un nâmus contre les ennemis). Le nâmus est contraire au bien public.

Figure 1. Les espaces sexues d'une maison traditionnelle

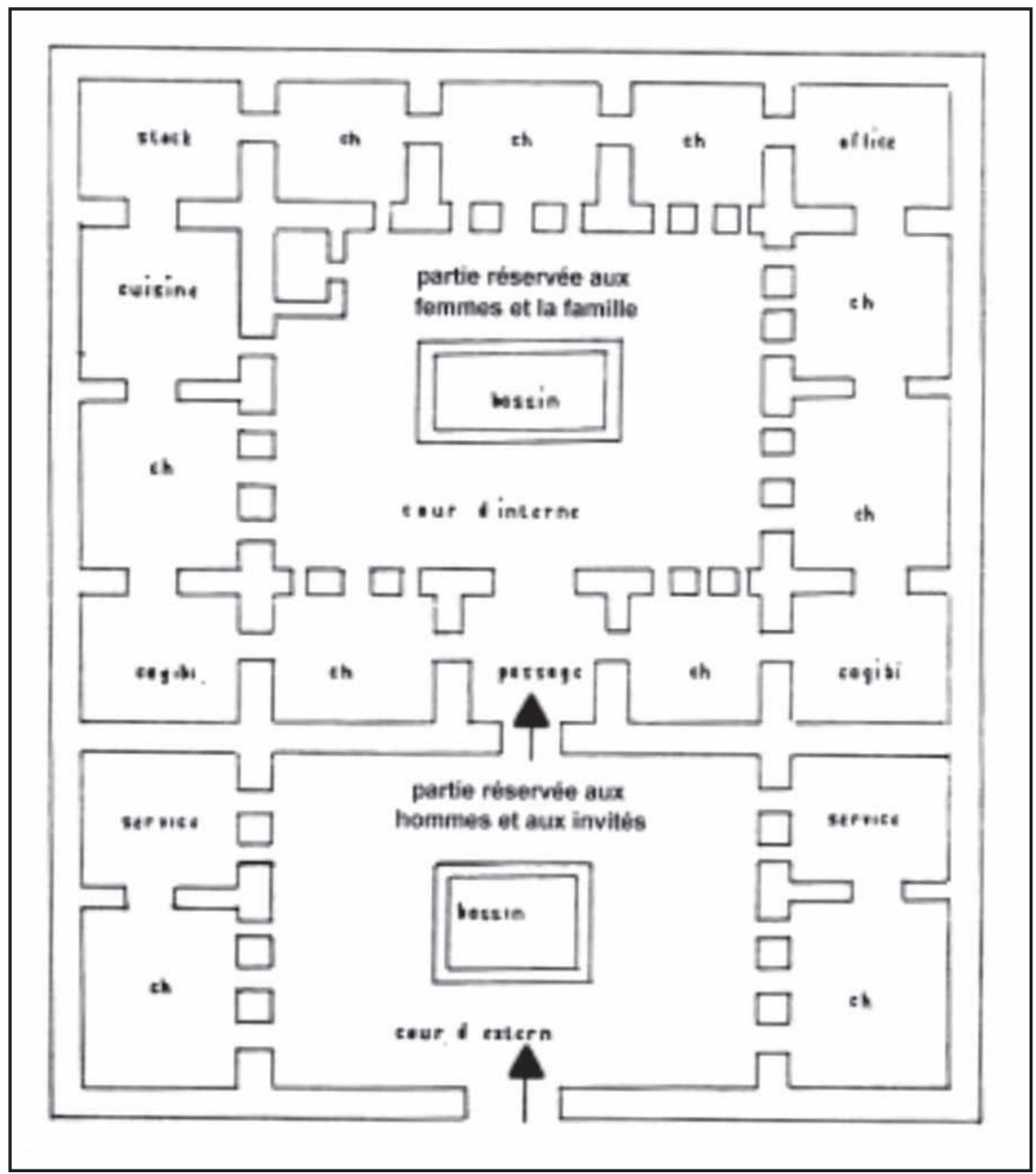

Source : Saidi-Shahrouz M., 1985. 
Depuis un demi-siècle, ce système est remis en question.

Dans les années 30, dans le cadre de ses projets de réforme et de modernisation de I'Iran, Réda Shah, le premier Roi Pahlavi obligea officiellement les femmes à abandonner le port du voile dans l'espace public. Des tchadors furent arrachés de façon souvent violente et de nombreuses manifestations furent réprimées. Cette émancipation autoritaire a soulevé la colère des traditionalistes et du clergé qui ont contribué à créer un mécontentement général et une forte opposition à la modernisation. Cette loi a eu un effet inverse. Les femmes ne sont plus sorties de leur maison. L'interdiction du port du voile fut levée en 1941.

A partir des années 60 , sous le règne de son fils Mohamad Réda Pahlavi, I'Iran a connu une modernisation spectaculaire. En ce qui concerne les femmes; elles ont acquis des droits politiques et juridiques par décret mais, en réalité, ce ne sont que l'élite et la classe moyenne instruite qui ont pu accéder à la sphère publique grâce à leur position sociale. Cette situation s'est projetée dans l'espace et la ville s'est divisée en deux parties : le nord "moderne » et riche et le sud populaire. Les femmes du sud restant invisibles dans les espaces urbains, tandis que les femmes des quartiers nord vivaient à I'occidentale et étaient bien visibles dans l'espace urbain.

Au lendemain de la révolution de 1979, L'Ayatollah Khomeyni, comprenant l'importance de l'implication des femmes peuples, les a appelées à manifester pour soutenir son régime. II leur a permis de jouer un rôle politique et a légitimé leur présence dans I'espace public (à la condition toutefois de respecter le " comportement islamique", notamment par le port obligatoire du hijâb). La généralisation du voile islamique, I'imposition de la ségrégation des sexes, dans les écoles ou les lieux publics, ont en revanche été vécues dans les milieux traditionnels comme des mesures sécurisantes, permettant aux femmes, surtout aux jeunes filles, d'avoir une activité publique sans enfreindre la morale sociale dominante. Comme le précise F. Adelkhah:

"Un père rigoriste n'avait plus d'arguments pour empêcher sa fille d'aller au lycée ou de prendre un petit emploi avant d'être mariée, comme convenu avec son cousin. Ce "tchadorpasseport " a ainsi permis aux femmes d'occuper au maximum l'espace public et surtout d'avoir accès à l'école, même en milieu rural $»^{1}$.

1. Adelkhah F., 1991, La Révolution sous le voile, Femmes islamiques d'Iran, Paris, Karthala, 280 p. 
Cependant, cette présence à l'extérieur a été possible, à condition pour les femmes de respecter certaines règles de conduite comme le port de la tenue islamique "le hijâb», la discrétion dans l'espace public, etc. Une autre condition, renvoyant aux traditions anciennes, est de ne jamais « séjourner » dans un lieu public mais de le traverser et d'être toujours en mouvement. Les rares archives photographiques du passé concernant les femmes à l'extérieur de leur maison, les montrent en marche ou prêtes à monter dans un carrosse, train ou automobile. C'est pourquoi la notion de mouvement a toujours été importante dans la représentation féminine en Iran. Plus précisément, nous pensons que les mobilités quotidiennes sont un prisme pertinent pour aborder la question de la condition féminine.

Aujourd'hui, selon les enquêtes de transport, plus d'un tiers des déplacements journaliers sont effectués par les femmes. Ce pourcentage est important si on tient compte du fait qu'elles ne sont que $12 \%$ à avoir un emploi salarié. Que signifie alors cette présence féminine massive et quotidienne dans les rues de Téhéran?

Afin de mieux comprendre le sens de cette féminisation de l'espace public, nous avons mené une enquête quantitative suivie d'entretiens qualitatifs, auprès d'un échantillon de 432 femmes résidant dans les 22 arrondissements de Téhéran. L'enquête comportait deux volets dont un cahier de bord qui a été remis à chaque femme sondée ${ }^{2}$ pour lui permettre de noter ses déplacements d'une manière précise, sur une durée d'une semaine. ${ }^{3}$ Pour chacun des déplacements effectués, il lui est demandé d'indiquer un ensemble d'informations : le jour, I'heure de sortie, la durée, le motif, le mode de déplacement, les personnes les accompagnant et la tenue vestimentaire portée. La fréquence relative à chacun des types de déplacements est demandée de manière à distinguer les déplacements à caractère exceptionnel des déplacements hebdomadaires, voire quotidiens. Ce recueil très précis a comptabilisé 2200 déplacements. Il a été complété par un questionnaire comprenant un ensemble de questions plus générales sur les activités pratiquées, le type de lieux fréquentés, les déplacements dans les villes voisines, etc. Enfin une série de questions sur leurs représentations de la ville et d'elles-mêmes dans la ville sont posées à la fin du questionnaire.

2. Bottai M. et Barsotti O., 1994, Lo spazio et la sua utilizzazione, Milan, Demos, FrancoAngeli, $219 \mathrm{p}$.

3. Une fois l'enquête réalisée, l'enquêteur laissait le carnet de bord qu'il venait récupérer une semaine plus tard. 
Les résultats de l'enquête montrent que les motifs de déplacement les plus fréquents sont les études et la formation ( $17 \%$ des trajets), puis viennent les achats journaliers et les loisirs (14\%). Le travail, lui, ne représente que $9 \%$ de l'ensemble des déplacements féminins.

Les résultats de l'enquête montrent aussi clairement la compétition entre deux sphères fondamentalement différentes. D'une part, les mobilités liées aux activités pour le maintien de la sphère familiale, traditionnelle, et de l'autre, les mobilités motivées par les activités de production de soi, projetées vers le futur.

Figure 2. Domaine familial, domaine individuel
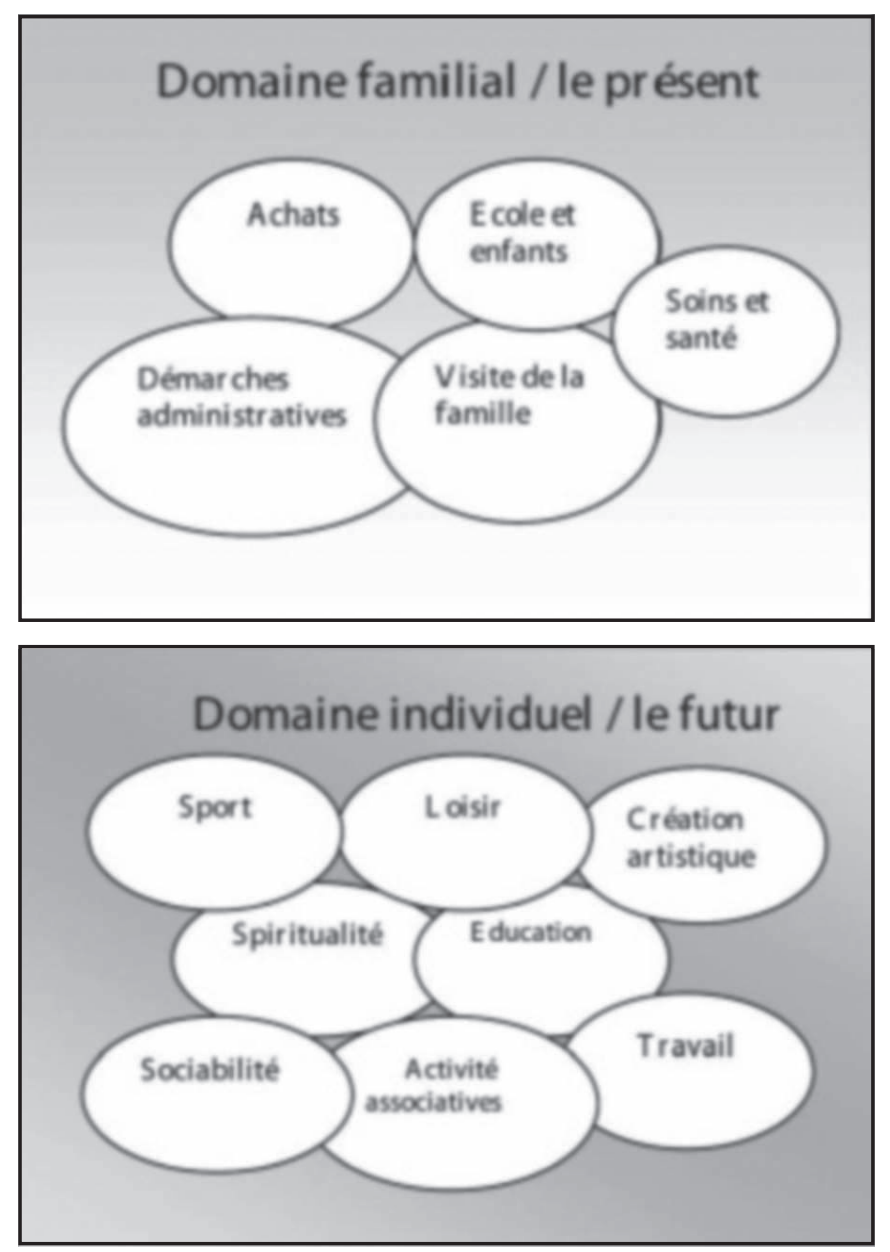

Source : Saidi-Shahrouz M., 2010. 
Les mobilités qui ont pour motif des activités liées à la famille représentent $44 \%$ de l'ensemble des mobilités et celles liées aux activités pour soi constituent $56 \%$ de l'ensemble des déplacements quotidiens. Ce constat nous démontre une nette supériorité des activités que les femmes entreprennent pour leur épanouissement personnel. Ceci peut être interprété comme un des signes de l'émergence de l'individu au féminin, bien que la question du faible taux de travail des femmes reste entièrement posée.

\section{Sortir pour se former, se cultiver et se détendre sous conditions}

Les déplacements pour les études et la formation, qui représentent au total une grande part des déplacements journaliers, s'effectuent majoritairement "seules". Ils reflètent à la fois la tendance croissante en Iran qu'ont les femmes à accéder à l'éducation et le changement profond que cela entraîne dans la société, notamment en matière de déplacements des femmes hors de leur domicile. On sait que celles-ci sont plus nombreuses que les garçons à se rendre à l'université. Le nombre de diplômées des universités publiques et privées a augmenté de $40 \%$ en 10 ans. Depuis l'année 2000 le taux de réussite à l'entrée à l'université est passé de $52 \%$ à $57 \%$ et depuis 2004 à $60 \%$ ce qui veut dire un million sept cent mille étudiantes contre quatre cent quatre-vingt-dix mille en 1988. Ceci confirme que, parallèlement à l'augmentation du nombre d'étudiantes, le nombre des filles dans les écoles, primaires et secondaires augmente aussi : en 1996 le taux de filles inscrites à l'école primaire était de $94,7 \%$, contre $75,5 \%$ en 1988 et le taux de filles inscrites dans les lycées et collèges est passé de $31 \%$ en 1988 à $59 \%$ en 1996 (Shadi Talab, 2004) .

Cet engouement pour les études et la formation montre à quel point les femmes sont conscientes que $c^{\prime}$ est par leurs compétences professionnelles qu'elles peuvent améliorer le statut des femmes et accéder au monde du travail et à la sphère publique.

En attendant les transformations sociales "visibles » et l'émancipation plus affirmée des femmes, le paysage urbain à Téhéran est devenu aujourd'hui le théâtre de nouveaux modes de vie et notamment dans les pratiques des espaces publics. Les lieux auparavant spécifiquement « masculins » sont investis par les familles toutes entières voire de plus

4. Shaidi Talab J., 2004, in "Zan va Farhang » S.DM. Mir Chokraï et A.R Hassan Zadeh Nashr-e Ney, Téhéran, 1018 p. 
en plus par les femmes seules. Par exemple, dans le domaine des sorties pour les loisirs, les femmes sont très inventives pour se trouver des activités au quotidien. Ces activités se classent en trois catégories dont la première correspond aux activités liées à la spiritualité qui se réalisent à la mosquée, au cimetière, dans les lieux de pèlerinage (mausolées). La deuxième rassemble les activités de sociabilité (réunion entre femmes, visites des amis, etc.) et enfin, la troisième renvoie aux loisirs au sens le plus moderne qui comprend les pratiques développées dans un "parc ", "à la montagne », " dans la ville », la fréquentation du " café », du " cinéma » et des « shopping centers».

Ces différentes catégories se superposent dans l'espace et dans le temps et ne peuvent être dissociées. Cette catégorisation apparaît très complexe lors des enquêtes. Compte tenu du poids des interdits imposés par les traditions et intériorisés par les femmes, avoir du bon temps, n'est pas facile à déclarer. A cela, il faut ajouter que, dans les espaces publics, le contrôle de l'État est institutionnalisé par la République islamique : les femmes sont donc particulièrement surveillées.

Le port du hijâb islamique est obligatoire en Iran pour sortir dans l'espace public, les femmes ont cependant un choix dans le degré d'acceptation de cette règle. En somme, toutes les femmes n'agissent pas de la même manière face à ce principe. La tenue la plus fréquemment portée lors des déplacements est le tchador (43\%), devant la tenue dite moderne composée souvent d'une veste longue et d'un petit foulard à la mode (31\%). Vient ensuite le port d'un "maghna-é », sorte de cagoule en tissu plutôt sombre (19\%). Nous avons noté au fil des chapitres précédents que la tenue varie de manière très significative selon les motifs de déplacement. Ainsi, pour les déplacements de visite à la famille, la part de déplacements effectués en tchador s'élève à $65 \%$, contre moins de $20 \%$ pour les déplacements qui correspondent à un motif professionnel. Le motif éducatif est le plus souvent associé au port de la cagoule "maghna-é » (52\%), alors que le motif loisirs coïncide le plus souvent avec une tenue "moderne ». Si la tenue principalement portée pour rendre visite à la famille est le tchador, il est aussi davantage porté dans les déplacements quotidiens de proximité (courses, accompagnement, enfants, école).

La cagoule "maghna-é », est davantage portée pour se rendre à l'école, à l'université ou dans tout autre lieu de formation. Elle est aussi adoptée pour se rendre à son travail (près de $40 \%$ des déplacements effectués en cagoule correspondent à un motif professionnel). Cela peut être comparé à un uniforme de travail. Le port d'une tenue moderne est surreprésenté dans les trajets qui ont pour objet une activité artistique ou sportive. En dehors de celles qui portent au quotidien 
la tenue moderne, certaines femmes, qui dans la journée se déplacent en cagoule "maghna-é », et manteau islamique, revêtent pour les activités de loisir, des tenues moins couvrantes (Saidi-Sharouz, 2010).

En dehors de la définition de la place de la femme par la société et l'État, il nous semble intéressant de comprendre ce que ressentent les femmes elles-mêmes quant à leur présence à l'intérieur? Quelles représentations ont-elles de la ville et des différents espaces publics?

Traditionnellement, en Iran, les femmes rentrent avant le coucher du soleil. Aujourd'hui, cette situation évolue et malgré les effets de dénonciation de la violence dans l'espace public, très présente dans la presse locale, nombreuses sont les femmes qui rentrent après la tombée de la nuit. Les entretiens menés montrent qu'elles ont de moins en moins peur de rester dehors et qu'elles sont prêtes à le faire si cela est nécessaire. Ainsi, il arrive fréquemment aux étudiantes, en raison de longs trajets effectués dans les transports en commun, de rentrer tard à la maison.

La géographie de l'insécurité dans la ville de Téhéran a changé. Si les femmes de plus de 50 ans que nous avons interrogées déclarent qu'elles avaient peur, avant la révolution, des quartiers sud et populaires, réputés pour leurs voyous aux « coups épais » (Adelkhah, 1998), leurs drogués et leurs tireurs de couteaux "tchaghou kesh", aujourd'hui, elles se sentent en insécurité partout. A la question: "Dans quels lieux de la ville vous vous sentez en insécurité ?", une partie des femmes répond qu'elles ressentent une insécurité dès qu'elles sortent de la maison. Mais il est intéressant de constater que cette peur est diffuse et ne correspond pas au seul risque d'agression dans un lieu bien défini, comme dans le passé. Elles expriment cette peur, autant par la probabilité de se faire contrôler par la police des mœurs, que d'être victimes d'un vol ou d'une agression. Elles disent également redouter la circulation automobile et les autoroutes désertes qui traversent certains quartiers. Malgré cela, à la question "Y-a-t-il des lieux où vous préféreriez être un homme pour vous y rendre? $»^{5}$, à l'exception de la pratique du sport à l'extérieur, elles disent ne pas avoir besoin d'être un homme pour se déplacer dans la ville. Ce qui prouve le sentiment d'une présence plutôt affirmée des

5. La question posée est la suivante : "Y-a- $t$-il des lieux où vous préféreriez être un homme pour vous y rendre? (transports en commun, pratique d'un sport dans un lieu public, lieux plus fréquentés par des hommes, nulle part, partout, autres)». 
femmes dans l'espace public.

\section{La diversité des femmes face à la mobilité}

Pour mettre en évidence et formaliser ces pratiques et perceptions spatiales nouvelles et très différenciées chez les femmes de Téhéran, nous avons cherché à mettre en relation l'ensemble des informations réunies par notre enquête sur les déplacements, pour créer un indicateur synthétisant les pratiques spatiales des femmes. Nous avons ainsi croisé les données concernant la durée cumulée des déplacements, les modalités dominantes de transport, la tenue vestimentaire, les motifs de déplacement, l'accompagnement, les perceptions, ce qui a permis d'identifier cinq types de mobilité des femmes, ou cinq profils de femmes en fonction de leur mobilitée .

- Les recluses dans l'espace privé » (22\% de l'échantillon des 400 p. 3 femmes). On constate que la moitié des femmes de cette catégorie semble avoir une sociabilité qui se réduit à celle du voisinage. La famille est peu présente et tient peu de place dans la vie de ces femmes, soit en raison d'un éloignement géographique, soit en raison de l'éloignement de la famille du conjoint. Ce profil de femmes traditionnelles se trouve surtout dans le centre ancien de Téhéran.

- Les mères de famille captives de la proximité » (34\%). Ces femmes dont la mobilité se réduit à des déplacements de proximité ou à des déplacements en famille, ne sont pas intégrées dans le monde de travail. Pour la plupart instruites, elles ne travaillent pas soit par choix soit par défaut d'un travail qui leur convienne. Ayant majoritairement plusieurs enfants, leur mobilité spatiale est de facto réduite à des déplacements de proximité.

- Les femmes actives, visibles dans la sphère professionnelle $(11 \%)$; Ces femmes ayant pour la plupart un emploi salarié fixe sont très minoritaires mais ce profil, dans les années qui viennent, peut devenir le profil type de la femme salariée et issue de la classe moyenne. Ce type de femmes, affranchie de l'espace géographique, pourrait résider dans n'importe quel quartier de la ville.

- Les jeunes femmes éduquées, aux pratiques spatiales très étendues (14\%); Les déplacements sont nombreux pour ces femmes dont un tiers sont étudiantes dans le supérieur, et près de la moitié travaille, le plus souvent dans le privé. De milieu social favorisé, elles ont un budget de transport élevé et se déplacent en voiture pour près de $90 \%$

6. Cette typologie a été réalisée avec le concours de l'INED où ont été traitées les données de l'enquête et le traitement statistique des données. Ce dernier a permis d'identifier et de définir cinq catégories de femmes. 
d'entre elles. Près de la moitié déclare ne jamais se déplacer à pied. Les loisirs sont aussi très présents dans la vie de ces femmes : trois-quarts d'entre elles fréquentent régulièrement les cafés et les cinémas. Elles se rendent au parc et rendent visite à leurs amis. La montagne est une destination très prisée pour plus de $60 \%$ de ces jeunes femmes : ne représente-t-elle pas un espace de liberté pour elles?

Cette population est très ouverte sur l'international et presque toutes les femmes maîtrisent, une langue étrangère et les deux tiers d'entre elles se sont déjà rendues à l'étranger. II n'existe pas de tenue spécifique pour ces femmes qui, le plus souvent, l'adaptent à leur activité. Elles peuvent porter la cagoule pour se rendre dans une administration publique, ou un simple foulard lorsqu'elles sortent avec des amis pour les loisirs.

- Les jeunes femmes modernes et visibles dans l'espace public (19 \%). Pour près de Trois-quarts d'entre elles, les motifs de déplacement sont liés aux études. La moitié déclare être étudiante. Les autres déplacements sont essentiellement liés aux loisirs : cinéma pour près de la moitié, quelques-unes fréquentent les cafés, d'autres regrettent de ne pas s'y rendre. Les raisons évoquées sont l'insécurité ou l'interdiction pour $20 \%$ d'entre elles. Par ailleurs, ces femmes pratiquent fréquemment des activités artistiques et sportives.

Si près de 90 \% déclarent parler une langue étrangère, ce n'est pas pour autant qu'elles se sont déjà rendues à l'étranger. Seules $18 \%$ de ces jeunes femmes ont déjà effectué un voyage à l'étranger. Les femmes de cette classe constituent la classe moyenne ascendante. Les mobilités fréquentes, les études et la connaissance d'une langue étrangère leur offrent une compétence supplémentaire et donc une meilleure estime de soi.

\section{CONCLUSION}

Cette classification n'enferme pas les femmes dans des catégories rigides. Elles ne sont pas isolées dans une société vivant avec ses traditions et où les relations inter-familiales, de voisinage etc., sont encore très fortes. C'est ainsi que les changements concernent tous les âges et toutes les classes sociales, comme si le mouvement des unes emportait les autres - moins mobiles - avec elles : la société féminine bouge à Téhéran et cherche à affirmer sa place. Cependant, elles savent qu'elles doivent relever des défis au quotidien et être inventives pour légitimer cette présence dans l'espace public. 
La sortie des femmes est un fait social et culturel aux conséquences profondes sur l'espace urbain. Cependant, cette liberté de mouvement n'est pas définitivement acquise. Des mécanismes de résistance se mettent régulièrement en place pour contrôler ce que certains considèrent comme un " débordement » en essayant de gérer cette présence au dehors : ex. : les pistes cyclables et parcs pour femmes, les entrées de bâtiment séparées pour femmes et hommes, les stades de foot réservés aux hommes, etc. sont des mesures mises en place au quotidien pour conserver la maîtrise par les hommes des espaces publics.

La "métropolisation » de Téhéran et les politiques urbaines favorisant la vision technocratique de la ville font partie d'autres menaces pour cette autonomie encore fragile. Très peu motorisées, les femmes se déplacent surtout à pied et dans les transports en commun. Peut-on croire que les mobilités des femmes dépendant fortement des modes de «transport doux » peuvent être viables si Téhéran choisit l'étalement urbain ? Les déplacements seraient sûrement plus difficiles et les femmes, moins motorisées seront particulièrement touchées par ces contraintes.

Les nuisances urbaines peuvent aussi devenir un obstacle à la mobilité féminine. Ceux qui engendrent le bruit, la pollution atmosphérique et les accidents de circulation et ceux qui en sont victimes ne sont pas les mêmes personnes, comme le rappelle Vincent Kaufmann ${ }^{7}$. Non seulement les personnes qui ne disposent pas d'un accès aisé à I'automobile se trouvent limitées dans leurs modes de vie, mais elles se trouvent par là même davantage exposées aux nuisances et aux dangers de la circulation. Si la société devient moins duale depuis 30 ans, elle reste cependant, profondément injuste. La «métropolisation " à "la californienne " accentue ces injustices. Le nouveau Schéma directeur de Téhéran actuellement en voie de finalisation, ne prend pas en compte la spécificité des conditions des femmes alors que le régime islamique donne à cette distinction de genre la place capitale que I'on sait. Cette contradiction ne semble guère faire débat en Iran. Les contraintes économiques et techniques liées à la mixité ou non-mixité dans les transports ne sont pas évoquées, pas plus que l'augmentation prévisible du nombre de femmes ayant une activité professionnelle permanente. Une fois qu'elles auront gagné le combat social et culturel pour l'accès à l'espace public, les femmes seront confrontées à des formes d'urbanisme et de services publics

7. Kaufmann V., 2000, Mobilité quotidienne et Dynamiques urbaines, Lausanne, Presses polytechniques et universitaires romandes, 252 p. 
inadaptées à leurs besoins. Mais en attendant, les femmes s'inventent leurs sorties et profitent d'être dehors pour se former, se cultiver, se détendre et tisser de nouveaux réseaux de sociabilité hors famille et tribu. Elles utilisent aussi ces sorties pour simplement être en dehors de chez elles et expérimenter la ville seules et d'une manière autonome. On peut donc leur reconnaître l'acquisition d'une « compétence » qui transforme peu à peu le devoir religieux et politique de l'époque révolutionnaire en un "droit à la ville».

\section{RÉFÉRENCES BIBLIOGRAPHIQUES}

- Adelkhah F., 1991, La Révolution sous le voile, Femmes islamiques d'Iran, Paris, Karthala, 280 p.

- Coutras J., 2003, Les Peurs urbaines et l'Autre Sexe, Paris, L'Harmattan, Collection logiques sociales, $242 \mathrm{p}$.

- 1996, Crise urbaine et espaces sexués, Paris, Armand Colin, 156 p.

- 1998, "La Mobilité quotidienne et les inégalités de sexe à travers le prisme des statistiques", dans Territoires, vol. 10, n², Québec, Recherches féministes Université Laval, p. 77-90.

- Denefle S., 2004, (dir.), Femmes et villes. Tours, Presses Universitaires Francois-Rabelais, Maisons des sciences de L'Homme «Villes et Territoires », $539 \mathrm{p}$.

- Göle N., 2003, Musulmanes et Modernes, voile et civilisation en Turquie, Paris, La Découverte, 190 p.

- HabiBi M., Hourcade B., 2005, Atlas de Téhéran métropole-Atlas of Tehran Métropolis-Atlas-E Kalânshahr-E Tehrân. Téhéran, Centre d'informations géographiques de Téhéran (TGIC), Vol 1. La terre et les hommes - Land and people - Sarzamin va mardom. Persan 218 p., Français 79 p., English 75 p., 214 Cartes.

- Hourcade B., 2004, "La recomposition des identités et des territoires en Iran islamique ", Annales de Géographie, n 638-639, p. 511-530.

- Kaufmann V., 2000, Mobilité quotidienne et Dynamiques urbaines, Lausanne, Presses polytechniques et universitaires romandes, $252 \mathrm{p}$.

- Kian-Thiebaut A., 1998, "La Formation d'une identité sociale féminine post-révolutionnaire : un enjeu de pouvoir ", dans Yavarid'Hellencourt N. (éd.) Les Femmes en Iran, Paris, L'Harmattan, p. 135-158.

- Saidi-Sharouz M., juin 2011, "Les mobilités quotidiennes des femmes à Téheran: entre visibilité et invisibilité, dans l'Espace Geographique. 
- Les femmes dans la ville, le 7 décembre 2010, Mobilités quotidiennes des femmes à Téheran. Ce doctorat soutenue à Nanterre - Paris la Défense.

- 1998, Évolution de l'habitat urbain à travers la transformation économique et politique en Iran, Diplôme d'architecture, École $\mathrm{d}^{\prime}$ architecture de Grenoble, $670 \mathrm{p}$.

- 2008, "Le Pique-nique en Iran, rituel festif et art de vivre ", dans le Pique-nique, ou l'éloge d'un bonheur ordinaire, sous la direction de F. Barthe-Deloizy, Bréal, p. 73-91.

- 2004, "Les mobilités quotidiennes des femmes à Téhéran : réalités et enjeux ", Femmes et Villes, sous la direction de S. Denèfle, Presse Universitaires François-Rabelais, Maison des Sciences de l'Homme, Villes et Territoires, $\mathrm{n}^{\circ}$ 8, p. 444-452.

- Shadi-Talab J., 2004, "Motalebat va entezaraté zanané Iran » (Les revendications des femmes iraniennes) in Zan va Farhang, sous la (dir.) de Mohamad Mir Chokraï et A.R Hassan Zadeh Nashr-e NeyTéhéran, p. 376-408. 
